\title{
Characterization of High-Fat, Diet-Induced, Non-alcoholic Steatohepatitis with Fibrosis in Rats
}

\author{
Zheng-Jie Xu $\cdot$ Jian-Gao Fan $\cdot$ Xiao-Dong Ding $\cdot$ \\ Liang Qiao · Guo-Liang Wang
}

Received: 8 November 2008/Accepted: 12 April 2009/Published online: 21 May 2009

(C) Springer Science+Business Media, LLC 2009

\begin{abstract}
An ideal animal model is necessary for a clear understanding of the etiology, pathogenesis, and mechanisms of human non-alcoholic steatohepatitis (NASH) and for facilitating the design of effective therapy for this condition. We aimed to establish a rat model of NASH with fibrosis by using a high-fat diet (HFD). Male Sprague-Dawley (SD) rats were fed a HFD consisting of $88 \mathrm{~g}$ normal diet, $10 \mathrm{~g}$ lard oil, and $2 \mathrm{~g}$ cholesterol. Control rats were fed normal diet. Rats were killed at 4, 8, 12, 16, 24, 36 , and 48 weeks after HFD exposure. Body weight, liver weight, and epididymal fat weight were measured. Serum levels of fasting glucose, triglyceride, cholesterol, alanine aminotransferase (ALT), free fatty acids (FFA), insulin, and tumor necrosis factor-alpha (TNF- $\alpha$ ) were determined. Hepatic histology was examined by H\&E stain. Hepatic fibrosis was assessed by VG stain and immunohistochemical staining for transforming growth factor beta 1 (TGF$\beta 1$ ), and alpha-smooth-muscle actin ( $\alpha$-SMA). The liver weight and liver index increased from week 4 , when hepatic steatosis was also observed. By week 8 , the body weight and epididymal fat weight started increasing, which
\end{abstract}

\section{Z.-J. Xu · J.-G. Fan · G.-L. Wang}

Center for Fatty Liver Disease, Shanghai First People's Hospital,

Jiao Tong University, Shanghai 200080, China

J.-G. Fan $(\bowtie) \cdot$ X.-D. Ding

Department of Gastroenterology, Xinhua Hospital,

Shanghai Jiao Tong University School of Medicine,

Shanghai 200092, China

e-mail: fanjiangao@gmail.com

L. Qiao $(\bowtie)$

Division of Gastroenterology and Hepatology,

Queen Mary Hospital, University of Hong Kong,

Hong Kong, China

e-mail: qiaol@hku.hk was associated with increased serum levels of FFA, cholesterol, and TNF- $\alpha$, as well as development of simple fatty liver. The serum ALT level increased from week 12. Steatohepatitis occurred from weeks 12 through 48. Apparent hepatic perisinosodial fibrosis did not occur until week 24, and progressed from week 36 to 48 with insulin resistance. Therefore, this novel model may be potentially useful in NASH study.

Keywords Non-alcoholic steatohepatitis - Fibrosis · Animal model $\cdot$ Rat

\section{Introduction}

Non-alcoholic steatohepatitis (NASH) is part of the spectrum of non-alcoholic fatty liver disease (NAFLD), currently the most common form of chronic liver disease in the United States and in many parts of the world [1-4]. NAFLD is a continuum of diseases characterized by macrovesicular hepatic fat accumulation alone (simple hepatic steatosis) or accompanied by signs of hepatocyte injury, mixed inflammatory cell infiltrate, and variable hepatic fibrosis in pericellular distribution (NASH); NASH may lead to cirrhosis and hepatocellular carcinoma (HCC) [4-6]. There is strong evidence that the prevalence of NAFLD worldwide has increased substantially over the past decades in parallel with global trends in over-nutrition (decreased physical activity with disproportionate high-fat food intake), obesity, type 2 diabetes mellitus (T2DM), and the metabolic syndrome (MetS) [1-4]. Present trends in the obesity and diabetes pandemic indicate that a further increase in NAFLD prevalence is likely in the near future [1].

However, very few effective therapeutic approaches for full-blown NAFLD/NASH are available up until this stage 
$[5,6]$. Development of efficient prevention and therapeutic options for NASH is hampered by a clear understanding of the etiology and mechanisms of this condition, which in turn is limited by a lack of a suitable study model [7, 8]. An animal model of NASH constitutes a very important approach to understanding the molecular mechanisms of NASH [9-11]. An in vivo NASH model in animals is also a very important method to test the therapeutic potency of certain agents against NASH [12-14]. NASH in animals can be produced by a wide variety of factors leading to changes in hepatic fat disposition. Some of these factors include over-nutrition, methionine and choline deficient (MCD) diet, physical inactivity, insulin resistance (IR), hepatotoxins, and deficiency of leptin and/or its receptors [15-30]. However, the currently available animal models of NASH are associated with various drawbacks in that they do not or only partially reflect the real pictures of human NASH in terms of etiology, pathogenesis, and disease mechanisms [8-11]. For example, spontaneous transition to significant steatohepatitis does not occur in nutritional and metabolic models of steatosis [8, 9]. Although a MCD diet induces typical changes of NASH in rodents [16, 17], and a 3-week ad libitum administration of a liquid high-fat diet (LHFD) also produces the typical pathology of NASH, including steatosis, hepatic inflammation, and fibrosis in Sprague-Dawley rats [31], these nutritional models do not reflect the diet features seen in NASH patients, as MCD is not commonly seen in human beings, and the MCD model can cause severe weight loss and cachexia, which are not characteristic of NASH seen in patients $[11,16,17]$. In addition to a lack of progressive pathology seen in typical NASH, the fatty acid composition of the LHFD model is vastly different from that of the diet of NASH patients in which a higher level of saturated fatty acid (SFA) and a low level of polyunsaturated fatty acid (PUFA) are typical $[18,19]$. The genetic rodent models of NASH also have limitations [7, 9, 29, 30]. For example, NASH models induced by deficiency of leptin and its receptors, although useful in assessing the biological significance of the gene involved, do not reflect the natural etiological background of NAFLD/NASH in patients and are thought to be resistant to the development of fibrosis. Pharmacological models of NASH are also frequently used, but they have a major limitation in not reflecting the obesity and IR, which are critically important in the development of human NASH. Obviously, all these models do not reflect the multi-factorial features of NASH observed in patients and thus are "non-physiological." Consequently, there is an outstanding need to establish NASH models that can best mimic the real pathogenesis and etiology of human NASH $[8,9]$.

In this study, we aimed to create a novel rat model of NASH by feeding the animals with a home-made high-fat diet (HFD), with the hope to reproduce the key features of human NASH for future research.

\section{Methods}

Animals and High-Fat Diet

Sprague-Dawley rats were purchased from Shanghai Experimental Animal Center of Chinese Academy of Sciences (Shanghai, China) and bred in an experimental animal unit of Shanghai First People's Hospital. All animals were allowed to acclimatize for a week on the normal diet before grouping. One hundred eighteen male rats weighing $140-160 \mathrm{~g}$ were then randomly divided into two groups: the HFD-fed group $(n=77)$ and control (pair-fed, PF) group $(n=41)$. The HFD group was prepared by mixing $2 \mathrm{~g}$ cholesterol and $10 \mathrm{~g}$ lard oil with $88 \mathrm{~g}$ normal pellet chow. The HFD was composed of the following energy sources: $52 \%$ was provided by carbohydrates, $30 \%$ by fat, and $18 \%$ by protein (overall calories: $4.8 \mathrm{kcal} / \mathrm{g}$ ). The PF rats were fed normal rat pellet chow, which contains $67 \%$ carbohydrates, $10 \%$ fat, and $23 \%$ protein as the energy sources (overall calorie: $3.6 \mathrm{kcal} / \mathrm{g}$ ). The composition of the diet used in this study and comparison with the MCD diet are summarized in Table 1. All rats were given humane care in a temperature-controlled environment with a 12:12-h light-dark cycle. They were housed in plastic cages in groups of four and were permitted ad libitum consumption of water and diet. The care and use of animals in this study was under the guidelines approved by the Animal Ethics Committee of the Shanghai Jiao-Tong University School of Medicine.

\section{Experimental Procedures}

All rats of both HFD-fed and PF groups were maintained at the above-mentioned conditions for 4-48 weeks. At the end of the time points $(4,8,12,16,24,36$, and 48 weeks after feeding with appropriate diet), animals were fasted

Table 1 Major components of the HFD and comparison with PFD and MCD diet (g/100 g diet)

\begin{tabular}{llrl}
\hline & PFD & HFD & MCD diet \\
\hline Carbohydrates & 60 & 52.8 & 65.5 \\
Cholesterol & 0 & 2.0 & 0 \\
Fat & 4 & 13.5 & 10 \\
Protein & 20 & 17.6 & 17.5 \\
Methionine & 0.78 & 0.69 & 0 \\
Choline & 0.125 & 0.11 & 0 \\
\hline
\end{tabular}

HFD high-fat diet, $P F D$ pair-fed diet, $M C D$ methionine and choline deficiency 
overnight and then killed by exsanguinations under light pentobarbital. The body weight was recorded. The blood samples were collected from the abdominal aorta and maintained at $-20^{\circ} \mathrm{C}$ until assayed. Liver was quickly removed and weighed. Part of the liver tissues was snap frozen in liquid nitrogen for RNA and protein analysis. A small piece of liver was immediately fixed in $10 \%$ neutralbuffered formalin for further histology analysis. The epididymal fat was removed and measured, and used as an indicator for visceral adipose tissue.

Serum Biochemistry and Tumor Necrosis Factor-Alpha $(\mathrm{TNF}-\alpha)$

Serum levels of fasting glucose, triglyceride, total cholesterol (TC), and alanine aminotransferase (ALT) were measured by a multichannel automatic analyzer (Bayer Advia 1650, Germany). Serum levels of free fatty acids (FFA) were assayed using a commercial kit (Wako Pure Chemical Industries, Ltd., Japan). Serum insulin levels were detected (only at week 12, 24, 36, and 48) by a radioimmunoassay-based commercial kit. TNF- $\alpha$ levels were measured by an ELISA-based commercial kit (Endogen, Inc., USA).

\section{Hepatic Pathological Evaluation}

For light microscopic analysis of liver histology, the paraffin-embedded liver tissues were cut into $4-\mu \mathrm{m}$ sections, and standard hematoxylin-eosin (H\&E) staining was performed. Hepatic fibrosis was assessed by Masson stain. Ten $200 \times$ light microscopic fields were viewed on each section and scored for the severity of hepatic steatosis, inflammation and fibrosis according to the following criteria [32]: For hepatic steatosis: grade 0, no fat; grade 1, steatosis occupying less than $33 \%$ of the hepatic parenchyma; grade $2,34-66 \%$ of the hepatic parenchyma; grade 3 , more than $66 \%$ of the hepatic parenchyma; for inflammatory cell infiltration: grade 0: none; grade 1, 1-2 foci/field; grade 2, 3-4 foci/field; grade 3, more than 4 foci/field. The staging of hepatic fibrosis was investigated by Masson staining as follows: 0 , none; 1 , mild, zone 3 , perisinusoidal; 2 , moderate, zone 3, perisinusoidal; 3 , portal/periportal; 4, bridging fibrosis [32].

Mitochondria of the liver were assessed by transmission electron microscopy (TEM), which as performed by the Electron Microscopy Center of the Shanghai Jiao Tong University School of Medicine.

Immunostaining of $\alpha$-SMA and TGF- $\beta 1$

Paraffin-embedded liver tissues were deparaffinized with xylene series, washed well with graded ethanol and permeabilized with $0.2 \%$ trypsin at room temperature for $30 \mathrm{~min}$. After inactivation of endogenous peroxidase by $3 \% \mathrm{H}_{2} \mathrm{O}_{2}$ for $20 \mathrm{~min}$, tissue sections were washed with phosphate-buffered saline (PBS) and reacted with appropriate primary antibodies at $4^{\circ} \mathrm{C}$ overnight. Rabbit anti-rat transforming growth factor-beta 1 (TGF- $\beta 1$ ) (Santa-Cruz Biotechnology Inc., Santa Cruz, CA) was diluted 100-fold in PBS. Mouse anti-rat alpha-smooth-muscle actin ( $\alpha$-SMA) (Sigma, Ltd., St Louis, MO) was diluted 200-fold in PBS. Negative control was included in each reaction with primary antibodies being replaced by PBS. Following primary antibody incubation, the tissue sections were washed in PBS and incubated with two-step appropriate secondary antibodies (anti-rabbit or anti-mouse, Antibody Diagnostica Inc., USA) for $30 \mathrm{~min}$ at room temperature. The tissue sections were then washed with PBS, reacted with $0.01 \%$ $\mathrm{H}_{2} \mathrm{O}_{2}$ and 3,3' diaminobenzidine (DAB; Dako, Denmark) at room temperature for 3-5 min, washed again with PBS and covered with glycerin gelatin for light microscopy. The immunostaining results were evaluated by an experienced hepatopathologists (XZJ) in at least five microscopic fields $(\times 200)$ of each section.

\section{Statistical Analysis}

Data were expressed as mean $\pm \mathrm{SD}$ unless otherwise specified. The Student's $t$ test was used to analyze individual differences. Rank samples were analyzed by the rank-sum test. Rate comparison was analyzed by the $U$ test. A value of $P<0.05$ was considered to be statistically significant.

\section{Results}

Effects of HFD on Body Weight, Visceral Obesity, and Liver Index

No rat died in either group during the whole experimental period. Body weight increased steadily from week 4 of both groups, but more significantly and progressively so in HFD-fed rats after week 16. Development of visceral obesity, as indicated by a progressive increase in epididymal fat weight, started as early as 4 weeks after HFD feeding. Along with the increased body weight and visceral adiposity, there was a marked increase in liver weight of HFD rats starting from week 4, leading to a progressively increased liver index (LI, calculated as liver weight/body weight $\times 100 \%$ ). The increase in liver weight, epididymal fat weight and LI was more significant in HFD-fed rats. These results are summarized in Table 2. 
Table 2 Body weight, epididymal fat weight, and liver indexes

\begin{tabular}{|c|c|c|c|c|c|c|c|c|}
\hline \multirow[t]{2}{*}{ Week } & \multicolumn{2}{|l|}{$n$} & \multicolumn{2}{|c|}{ Body weight (g) } & \multicolumn{2}{|c|}{ Epididymal fat (g) } & \multicolumn{2}{|c|}{ Liver index (\%) } \\
\hline & PFD & HFD & PFD & HFD & PFD & HFD & PFD & HFD \\
\hline 4 & 6 & 12 & $329.2 \pm 12.7$ & $332.9 \pm 26.6$ & $8.5 \pm 1.5$ & $9.7 \pm 1.5$ & $2.5 \pm 0.1$ & $3.1 \pm 0.1^{*}$ \\
\hline 8 & 6 & 12 & $439.2 \pm 33.1$ & $435.8 \pm 26.7$ & $10.3 \pm 1.2$ & $12 \pm 1.2 * *$ & $2.8 \pm 0.1$ & $3.4 \pm 0.5^{* *}$ \\
\hline 12 & 6 & 12 & $450.8 \pm 50.6$ & $458.8 \pm 28.3$ & $12.1 \pm 2.3$ & $14.5 \pm 2.0^{*}$ & $2.1 \pm 0.2$ & $3.5 \pm 0.2 * *$ \\
\hline 16 & 6 & 12 & $478.2 \pm 36.8$ & $505.8 \pm 60.1$ & $14.0 \pm 1.8$ & $17.4 \pm 0.7 * *$ & $2.3 \pm 0.3$ & $3.9 \pm 0.5^{* *}$ \\
\hline 24 & 5 & 9 & $493.3 \pm 51.1$ & $549.9 \pm 50.0^{*}$ & $14.8 \pm 2.3$ & $19.9 \pm 2.1^{* *}$ & $2.3 \pm 0.3$ & $4.0 \pm 0.1 * *$ \\
\hline 36 & 6 & 10 & $506.3 \pm 47.2$ & $575.4 \pm 45.5^{*}$ & $15.4 \pm 2.4$ & $21.3 \pm 4.4^{* *}$ & $2.2 \pm 0.2$ & $4.2 \pm 0.3 * *$ \\
\hline 48 & 6 & 10 & $511.4 \pm 56.8$ & $591.94 \pm 76.7 *$ & $15.6 \pm 2.7$ & $24.4 \pm 7.4 * *$ & $2.2 \pm 0.3$ & $4.4 \pm 0.5^{* *}$ \\
\hline
\end{tabular}

Liver index calculated as liver weight/body weight $\times 100 \%$

$H F D$ high-fat diet, $P F D$ pair-fed diet

$* P<0.05$ vs. $\mathrm{PF}, * * P<0.01$ vs. $\mathrm{PF}$

\section{Serum Lipids, ALT, FFA, and TNF- $\alpha$}

The serum concentration of TC increased significantly from week 12 and continued to increase thereafter in HFDfed rats. The serum concentration of triglycerides varied during the study course, and no significant difference between the two groups was observed. Compared with PFfed controls, the serum ALT concentration increased from week 12 to week 48, and peaked at week 16 in HFD rats.

Relative to PF-fed rats, the serum concentration of FFA increased progressively from week 4 through week 48 in HFD-fed rats, which is associated with a significant and progressive increase in the serum TNF- $\alpha$, which peaked between weeks 12 and 16 . The serum glucose levels did not increase until week 36 . The above results are summarized in Table 3.

\section{HOMA-IR Index}

The HOMA-IR index of each group was calculated as follows: (fasting serum glucose $\times$ fasting serum insulin)/ 22.5. At both 12 and 24 week, the IR index was similar in the two groups. From week 36 to week 48, the IR index increased significantly in HFD-fed rats (Table 4).

\section{Hepatic Steatosis and Inflammation}

Overall, no abnormalities were observed in the liver tissues of the PF-fed control rats both macroscopically and microscopically. In contrast, livers from HFD-fed rats were generally enlarged with a yellowish and greasy appearance and a crisp texture.

Under the light microscope, livers of HFD-fed rats at week 4 showed diffusely mixed hepatic steatosis, which was predominantly microvesicular and mainly involved zone 3 hepatocytes (Fig. 1). Steatosis advanced to a more severe degree after 8 weeks of HFD feeding. At this time point, 11 out of 12 rats developed grade 1 or 2 hepatic steatosis, and mild hepatic lobular inflammation was present (Fig. 1).

After 12 weeks of HFD feeding, all rats developed hepatic changes consistent with moderate to severe stetaosis. Hepatic inflammation was more severe than those in HFD-fed rats at weeks 4 and 8 (Table 5). All liver samples showed moderate to severe lobular inflammation and focal necrosis. Both acute and chronic hepatic inflammation was present (as demonstrated by infiltration with polymorphs and mononuclear cells, respectively). However, only mild inflammation was observed in the portal zone at this stage (Fig. 1).

An even greater degree of hepatic steatosis and lobular inflammation was observed by week 16 (Table 5). Apart from infiltration with numerous polymorphs and mononuclear cells in hepatic lobules, intervening necrotic foci were frequently observed. Portal inflammation was also noted (Fig. 1).

From week 24 to week 48 , moderate to severe hepatic steatosis in HFD-fed rats was still present, but the lobular and portal inflammation was similar to that of the week 12 and week 16 groups (Table 5; Fig 1).

\section{Hepatic Fibrosis}

Hepatic fibrosis was assessed by Masson stain, immunostaining $\alpha$-SMA and TGF- $\beta 1$ in the harvested liver tissues. No histological signs of hepatic fibrosis were observed in PF-fed controls rats. By Masson stain, obvious hepatic fibrosis was not observed at weeks 4, 8, and 12 in HFD-fed rats. At week 16, mild perisinusodial fibrosis was noted in four rats. At week 24, all rats developed preisinusoidal fibrosis in zone 3. Perivenular and periportal fibrosis also was present (Table 5). At weeks 36 and 48, the hepatic fibrosis was more severe. Bridging fibrosis was observed in 
some rats. The representative staining results of week 24 , 36, and 48 livers are shown in Fig. 2.

In the PF-fed control livers, $\alpha$-SMA, and TGF- $\beta 1$ were localized along the blood vessels and occasionally on the sinusoidal walls. In the livers of week 4 and 8 HFD-fed rats, weak $\alpha$-SMA staining was observed on the sinusoidal wall, but the hepatic expression of TGF- $\beta 1$ was similar to that of the PF-fed control rats. From weeks 12 through 48, progressively increased expression of $\alpha$-SMA and TGF- $\beta 1$ were observed. Cells stained positive for $\alpha$-SMA were largely localized along the sinusoidal wall, in inflammatory cells and in the areas with remarkable perisinusoidal fibrosis. Positive staining of TGF- $\beta 1$ had similar histological distribution, except it was occasionally observed in hepatocytes (Fig. 3).

\section{Changes of Mitochondria}

At the ultrastructural level, electron microscopy was performed to assess the changes of mitochondria. Degenerative changes, including swelling, rounding up and loss of cristae were observed in the livers of HFDfed rats. Impairment of mitochondria became increasingly severe as the rats were exposed to HFD for a longer period of time. Stacks of intramitochondrial paracrystalline inclusion bodies were noted in some mitochondria (Fig. 4).

\section{Discussion}

Medical research is often constrained by the limited availability of both healthy and diseased tissue, making animal models an essential tool for studies of disease pathogenesis. Ideally, animal models should not only reproduce the established pathological expression of the human disease, but also recapitulate the context within which a disease develops and progress [11]. These issues are pertinent to hepatology research, in particular the study of NASH and its consequences. To date, there is no single animal model that displays the range of histopathologic and pathophysiologic features associated with NASH [7-9, 31]. Currently available models either mimic the histopathologic features of NASH or the pathogenic correlates of human NASH. The majority of animal models with IR are defective in leptin or adiponectin signaling and do not develop steatohepatitis spontaneously with the significant levels of fibrosis seen in patients. Animal models of fibrosing steatohepatitis usually are under high levels of oxidant stress as a result of increased hepatic fat load in the setting of reduced levels of antioxidants, resulting in lipotoxicity. These models usually do not exhibit 
Table 4 Serum glucose, insulin, and IR index

\begin{tabular}{|c|c|c|c|c|c|c|}
\hline \multirow[t]{2}{*}{ Week } & \multicolumn{2}{|c|}{ Glucose $(\mathrm{mmol} / \mathrm{l})$} & \multicolumn{2}{|l|}{ Insulin $(\mathrm{pmol} / \mathrm{l})$} & \multicolumn{2}{|l|}{ IR index } \\
\hline & PFD & HFD & PFD & HFD & PFD & HFD \\
\hline 12 & $5.2 \pm 1.5$ & $5.4 \pm 1.8$ & $98.03 \pm 14.07$ & $96.40 \pm 12.90$ & $23.72 \pm 6.99$ & $23.34 \pm 7.61$ \\
\hline 24 & $5.4 \pm 1.8$ & $5.6 \pm 2.0$ & $103.58 \pm 18.10$ & $95.11 \pm 27.78$ & $25.21 \pm 9.19$ & $24.87 \pm 9.48$ \\
\hline 36 & $6.2 \pm 1.7$ & $10.1 \pm 1.6^{*}$ & $97.34 \pm 16.35$ & $107.95 \pm 22.24$ & $27.38 \pm 8.62$ & $48.07 \pm 11.02^{*}$ \\
\hline 48 & $6.4 \pm 1.9$ & $10.5 \pm 2.6^{*}$ & $94.46 \pm 18.22$ & $99.98 \pm 23.09$ & $27.41 \pm 8.17$ & $45.49 \pm 11.36^{*}$ \\
\hline
\end{tabular}

HOMA-IR index $=($ glucosue $\times$ insulin $) / 22.5$

In PF groups at weeks $4,8,12$, and $16, n=6$; at week $24, n=5$

In HFD groups at weeks $4,8,12$, and $16, n=12$; at week $24, n=9$

$H F D$ high-fat diet, $P F D$ pair-fed diet

$* P<0.05$ vs. PFD
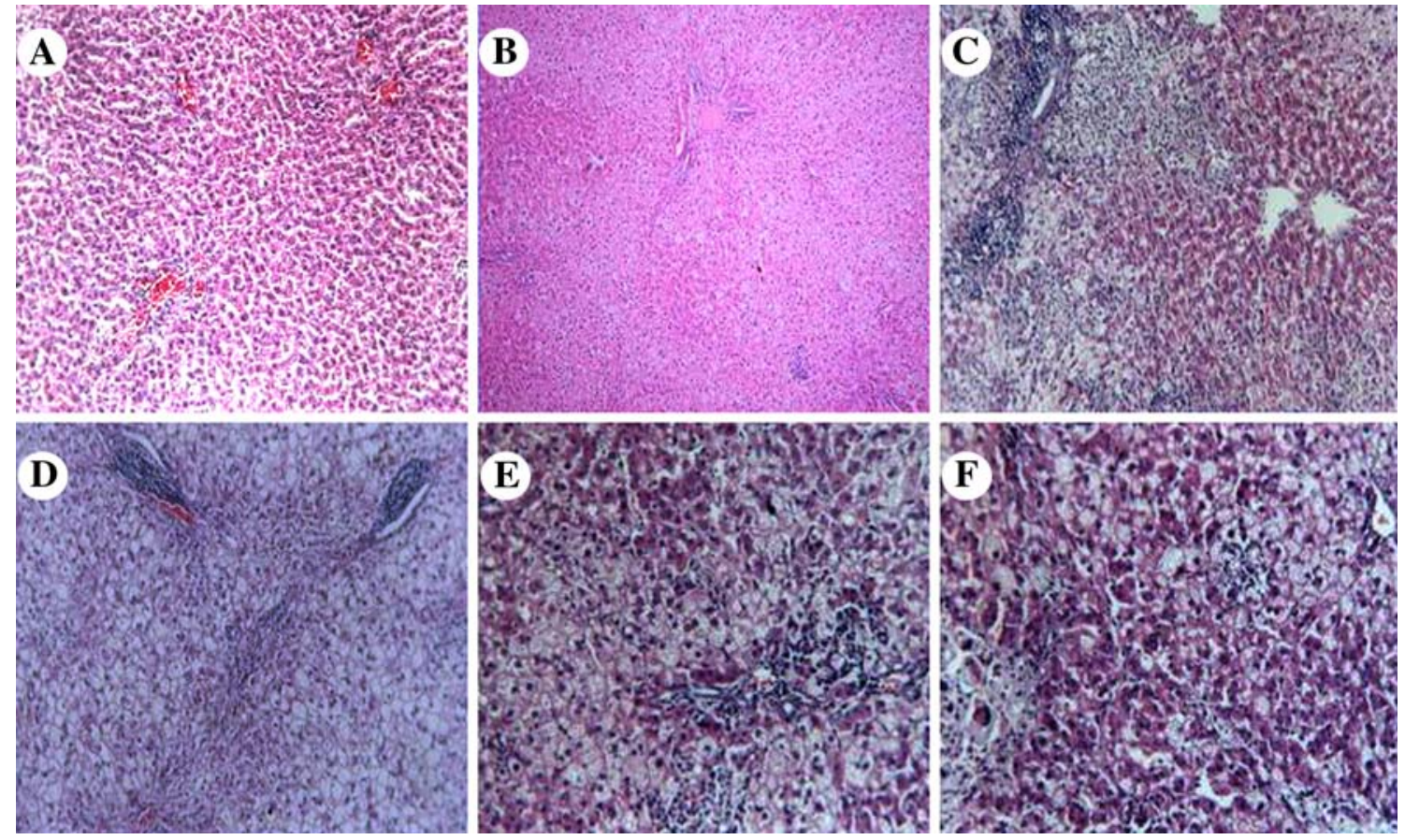

Fig. 1 H\&E stain of liver tissues from control (a), HFD-fed livers at weeks 8 (b), 16 (c), 24 (d), 36 (e), and 48 (f). Original magnifications: $\times 100(\mathbf{a}-\mathbf{d}), \times 200(\mathbf{e}, \mathbf{f})$

peripheral IR and have other limitations, such as excessive weight loss, usually not seen in the human disorder $[8,9$, $11,31]$. Therefore, a practically simple model that can best mimic the clinical aspects of NASH is very necessary. In this study, we produced a HFD by adding $10 \%$ (w/w) lard oil and $2 \%(\mathrm{w} / \mathrm{w})$ cholesterol to the normal pellet chow. Following 48 weeks of exposure, all experimental rats developed hepatic changes typical of human NASH. Based on several aspects described below, we considered our model a practical and accurate one for studying NASH.

In our model, NAFLD/NASH was successfully reproduced in all rats chronically fed with HFD. Grade 1 hepatic steatosis (i.e., steatosis of less than 33\% of the hepatic parenchyma) was observed at week 4 , and fatty liver (steatosis of more than $33 \%$ of the hepatic parenchyma) developed by week 8 . By week 12 through week 48 , steatohepatitis developed, and abnormal liver function occurred.

As development of hepatic fibrosis is one of the clinical features of late stage NAFLD (type 4), we investigated whether fibrosis occurred in our model. Masson stain revealed that a small percentage of rats developed mild perisinosodial fibrosis by week 16 . At week 24 , all the rats developed perisinosodial fibrosis. At week 36 and 48, the 
Table 5 Scores of hepatic steatosis, inflammation, and fibrosis staging

\begin{tabular}{|c|c|c|c|c|c|c|c|c|c|}
\hline \multirow[t]{2}{*}{ Group } & \multirow[t]{2}{*}{ Week } & \multirow[t]{2}{*}{$n$} & \multirow[t]{2}{*}{ Steatosis score } & \multirow[t]{2}{*}{ Inflammation score } & \multicolumn{5}{|c|}{ Fibrosis stage $(n)$} \\
\hline & & & & & 0 & 1 & 2 & 3 & 4 \\
\hline PFD & & 41 & 0 & 0 & 29 & 0 & 0 & 0 & 0 \\
\hline \multirow[t]{7}{*}{ HFD } & 4 & 12 & $1.67 \pm 0.79 *$ & 0 & 12 & 0 & 0 & 0 & 0 \\
\hline & 8 & 12 & $2.33 \pm 0.65^{*}$ & $0.3 \pm 0.24$ & 12 & 0 & 0 & 0 & 0 \\
\hline & 12 & 12 & $2.75 \pm 0.45^{*}$ & $1.70 \pm 0.77^{*}, \mathrm{a}$ & 12 & 0 & 0 & 0 & 0 \\
\hline & 16 & 12 & $2.91 \pm 0.29 *$ & $2.57 \pm 0.71^{*}, \mathrm{~b}$ & 8 & 4 & 0 & 0 & 0 \\
\hline & 24 & 9 & $2.67 \pm 0.50^{*}$ & $1.82 \pm 0.60^{*, \mathrm{c}}$ & 0 & 3 & 3 & 2 & 1 \\
\hline & 36 & 10 & $2.71 \pm 0.61^{*}$ & $1.93 \pm 0.73^{*, c}$ & 0 & 2 & 4 & 2 & 2 \\
\hline & 48 & 10 & $2.65 \pm 0.58^{*}$ & $1.91 \pm 0.69^{*, \mathrm{c}}$ & 0 & 1 & 4 & 3 & 2 \\
\hline
\end{tabular}

In PFD groups at weeks 4, 8, 12, and 16, $n=6$; at week $24, n=5$

In HFD groups at weeks $4,8,12$, and $16, n=12$; at week $24, n=9$

$H F D$ high-fat diet, $P F D$ pair-fed diet

$* P<0.05$ vs. PFD group

${ }^{\text {a }} P<0.05$ vs. weeks 4 and 8 HFD group

b $P<0.05$ vs. week 12 HFD group

${ }^{c} P<0.05$ vs. week 16 HFD group

Fig. 2 Masson stain was performed to detect fibrosis of liver tissues from control (a) and HFD-fed liver at week 24 (b), 36 (c), and 48 (d). Original magnifications: $\times 200$

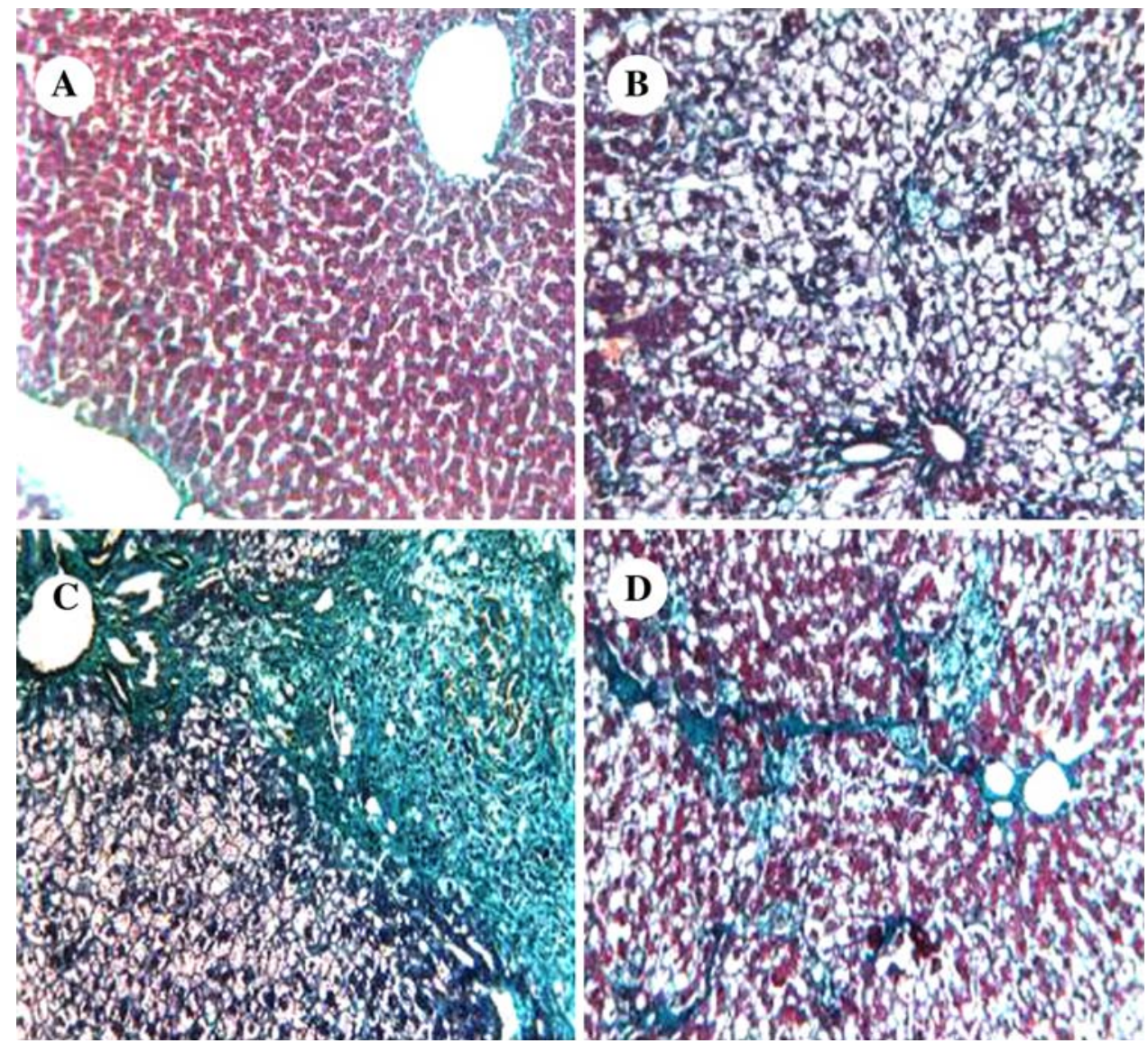

hepatic fibrosis was more severe. Bridging fibrosis was observed in some rats. The observed sequence of events (simple fatty liver, steatohepatitis, and steatohepatitis with progressive fibrosis) in our experimental rats closely mimics that of human spectrum of NASH. Further, these pathological features were predominant in zone 3. The lobular inflammation was more severe than portal inflammation. At the early stage, fibrosis had more of a 


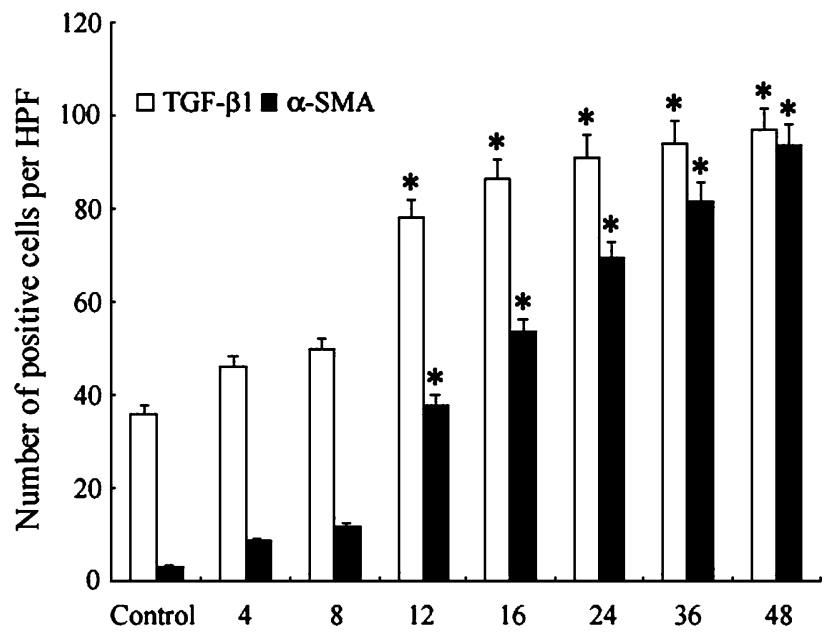

Fig. 3 Effect of HFD on hepatic expression of TGF- $\beta 1$ and $\alpha$-SMA. Immunostaining was performed as described in "Materials and Methods;" positively stained cells were counted in five randomly chosen high-power fields (HPF) and expressed as the average number of positive stain cells per HPF. $* P<0.05$, vs. control, week 4 and week 8 of respective groups. The representative results are shown perisinosodial pattern. The fact that the severity of hepatic steatosis and inflammation was not compatible with that of fibrosis suggests that the observed pathological changes closely mimic the pathological changes observed in human "cryptogenic cirrhosis" [4, 6]. Another pathological feature reproduced by this model is the presence of mitochondrial lesions such as swelling, rounding up and loss of crista. These changes are frequently observed in human $\mathrm{NASH}$ and are thought to be mechanistically linked to this condition [7, 8, 28, 33].

NAFLD/NASH is now considered one of the hepatic manifestations of MetS [1-5], which should ideally be reflected in animal models of NASH. In particular, increased serum levels of FFA and TNF- $\alpha$ are believed to be the two key factors in the pathogenesis of IR and MetS $[3,6]$. In our model, HFD-fed rats developed NAFLD, visceral obesity and hyperlipidaemia after 24 weeks of exposure. In addition, these rats also exhibited increased serum levels of FFA and TNF- $\alpha$ by week 24. These changes are mechanistically linked to the development of
Fig. 4 Electron microscopy of hepatic mitochondria in control (a), week 12 (b), week 16 (c), and week 24 (d) of HFD groups. Magnifications: a, b: $\times 16,800$; c: $\times 9,600 ; \mathbf{d}: \times 7,600$
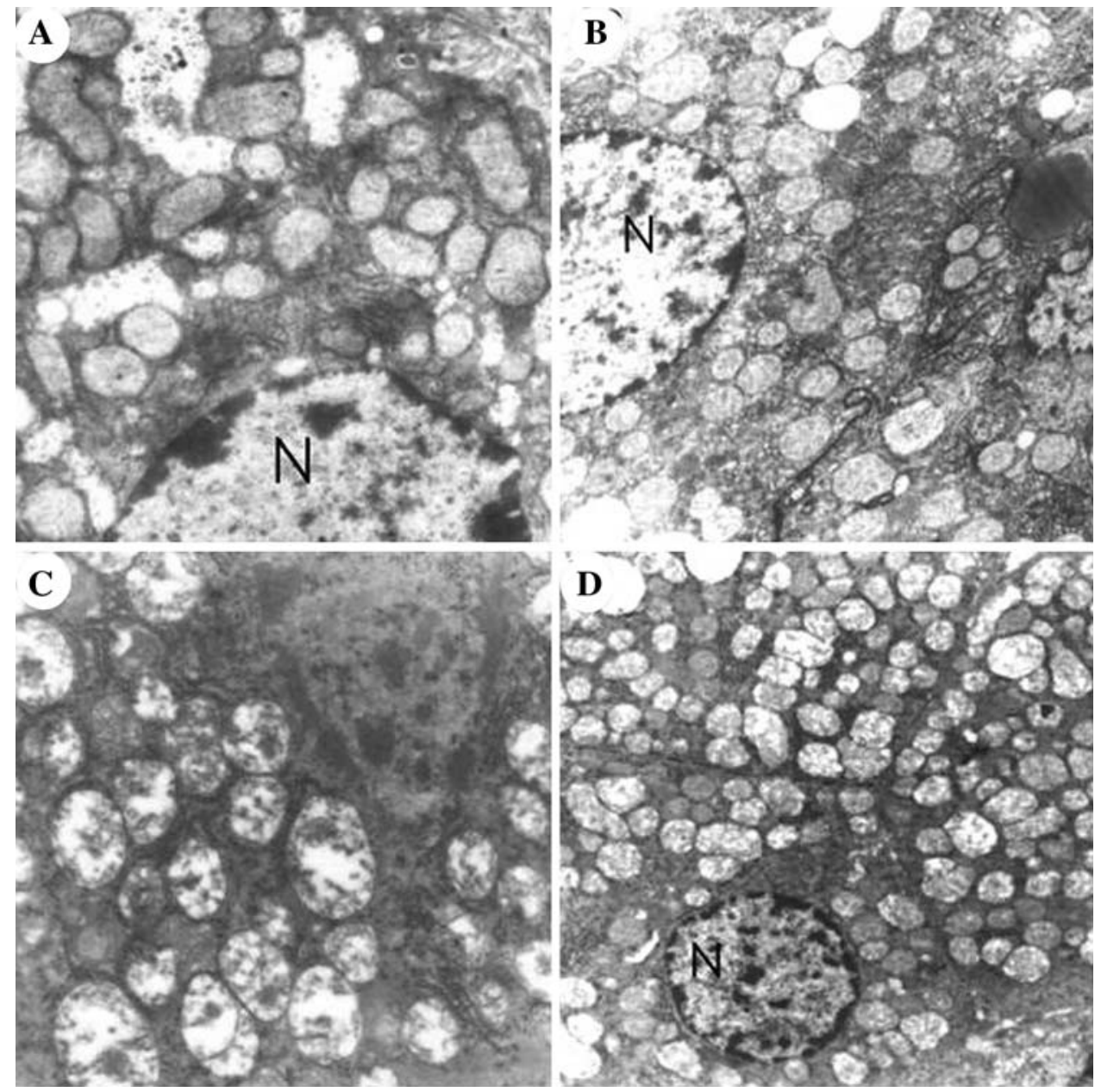
IR and MetS [7-9]. Conventional concepts hold that visceral obesity and IR, mostly mediated by adipokines such as TNF- $\alpha$, result in increased FFA release from visceral adipose tissue with the consequent enhancement of lipid delivery to the liver. Furthermore, FFA can stimulate $\mathrm{TNF}-\alpha$ expression in hepatocytes and in adipocytes, and thus is closely implicated in the etiology of IR [7, 34].

From week 36 to week 48, HFD-fed rats developed hyperglycemia and increased IR index. The appearance of these phenomena was later than that of NAFLD and visceral obesity. We think our current model mimics the real process of human MetS. According to the well-known "single gateway hypothesis," hepatic steatosis and hepatic IR are early events that usually precede pancreas insulin secretion decompensation and hyperglycemia [34]. The connection between NASH and hyperglycemia is indicated by the epidemiological finding of others that NAFLD significantly increases the risk of T2DM and cardiovascular complications, thus making NAFLD a pro-diabetic/proatherogenic state $[3,5,34,35]$. Therefore, we believe that the HFD in our study induces changes of NASH that mimic almost all clinical aspects of human NASH, depending on the experimental design.

In conclusion, we created a practically simple but accurate rat NASH model. It reproduced the pathological sequence of events typical of human NASH. This model also displays some of the features of MetS, such as increased visceral obesity, hyperlipidemia, increased FFA, and TNF- $\alpha$. The HFD can be easily home-made with good safety and low cost. Thus, our model may serve as an ideal tool to study the pathogenesis of NASH. However, further animal studies are warranted before this model can be widely promoted in NASH study.

\section{References}

1. Williams R. Global changes in liver disease. Hepatology. 2006;44:521-526. doi:10.1002/hep.21347.

2. Angulo P. GI epidemiology: nonalcoholic fatty liver disease. Aliment Pharmacol Ther. 2007;25:883-889.

3. Fan JG, Farrell GC. Epidemiology of non-alcoholic fatty liver disease in China. J Hepatol. 2009;50:204-210. doi:10.1016/ j.jhep.2008.10.010.

4. Ong JP, Younossi ZM. Epidemiology and natural history of NAFLD and NASH. Clin Liver Dis. 2007;11:1-16. doi:10.1016/ j.cld.2007.02.009.

5. Fan JG, Saibara T, Chitturi S, et al. What are the risk factors and settings for non-alcoholic fatty liver disease in Asia-Pacific? $J$ Gastroenterol Hepatol. 2007;22:794-800. doi:10.1111/j.14401746.2007.04952.x.

6. Farrell GC, Larter CZ. Nonalcoholic fatty liver disease: from steatosis to cirrhosis. Hepatology. 2006;43:S99-S112. doi: 10.1002/hep.20973.

7. Wanless IR, Shiota K. The pathogenesis of nonalcoholic steatohepatitis and other fatty liver diseases: a four-step model including the role of lipid release and hepatic venular obstruction in the progression to cirrhosis. Semin Liver Dis. 2004;24:99-106. doi: $10.1055 / \mathrm{s}-2004-823104$.

8. London RM, George J. Pathogenesis of NASH: animal models. Clin Liver Dis. 2007;11:55-74. doi:10.1016/j.cld.2007.02.010.

9. Nanji AA. Animal models of nonalcoholic fatty liver disease and steatohepatitis. Clin Liver Dis. 2004;8:559-574. doi:10.1016/ j.cld.2004.04.002.

10. Nanji AA. Another animal model for nonalcoholic steatohepatitis: how close to the human condition? Am J Clin Nutr. 2004; 79:350-351.

11. Larter CZ. Not all models of fatty liver are created equal: understanding mechanisms of steatosis development is important. J Gastroenterol Hepatol. 2007;22:1353-1354. doi:10.1111/ j.1440-1746.2007.05004.x.

12. Uno M, Kurita S, Misu H, et al. Tranilast, an antifibrogenic agent, ameliorates a dietary rat model of nonalcoholic steatohepatitis. Hepatology. 2008;48:109-118. doi:10.1002/hep.22338.

13. Fan JG, Xu ZJ, Wang GL. Effect of lactulose on establishment of a rat non-alcoholic steatohepatitis model. World J Gastroenterol. 2005;11:5053-5056.

14. Shen B, Yu J, Wang S, et al. Phyllanthus urinaria ameliorates the severity of nutritional steatohepatitis both in vitro and in vivo. Hepatology. 2008;47:473-483. doi:10.1002/hep.22039.

15. Svegliati-Baroni G, Candelaresi C, Saccomanno S, et al. A model of insulin resistance and nonalcoholic steatohepatitis in rats: role of peroxisome proliferator-activated receptor-alpha and n-3 polyunsaturated fatty acid treatment on liver injury. Am J Pathol. 2006;169:846-860. doi:10.2353/ajpath.2006.050953.

16. Sundaram SS, Whitington PF, Green RM. Steatohepatitis develops rapidly in transgenic mice overexpressing Abcb11 and fed a methionine-choline-deficient diet. Am J Physiol Gastrointest Liver Physiol. 2005;288:G1321-G1327. doi:10.1152/ajpgi.004 55.2004.

17. Kashireddy PV, Rao MS. Lack of peroxisome proliferator-activated receptor alpha in mice enhances methionine and choline deficient diet-induced steatohepatitis. Hepatol Res. 2004;30:104110. doi:10.1016/j.hepres.2004.06.004.

18. Musso G, Gambino R, De Michieli F, et al. Dietary habits and their relations to insulin resistance and postprandial lipemia in nonalcoholic steatohepatitis. Hepatology. 2003;37:909-916. doi: 10.1053/jhep.2003.50132.

19. Romestaing C, Piquet MA, Bedu E, et al. Long term highly saturated fat diet does not induce NASH in Wistar rats. Nutr Metab (Lond). 2007;21(4):4. doi:10.1186/1743-7075-4-4.

20. Baumgardner JN, Shankar K, Hennings L, et al. A new model for nonalcoholic steatohepatitis in the rat utilizing total enteral nutrition to overfeed a high-polyunsaturated fat diet. Am J Physiol Gastrointest Liver Physiol. 2008;294:G27-G38. doi: 10.1152/ajpgi.00296.2007.

21. Matsuzawa N, Takamura T, Kurita S, et al. Lipid-induced oxidative stress causes steatohepatitis in mice fed an atherogenic diet. Hepatology. 2007;46:1392-1403. doi:10.1002/hep.21874.

22. Savransky V, Bevans S, Nanayakkara A, et al. Chronic intermittent hypoxia causes hepatitis in a mouse model of dietinduced fatty liver. Am J Physiol Gastrointest Liver Physiol. 2007;293:G871-G877. doi:10.1152/ajpgi.00145.2007.

23. Nakayama H, Otabe S, Ueno T, et al. Transgenic mice expressing nuclear sterol regulatory element-binding protein $1 \mathrm{c}$ in adipose tissue exhibit liver histology similar to nonalcoholic steatohepatitis. Metabolism. 2007;56:470-475. doi:10.1016/j.metabol.2006. 11.004.

24. Kitade M, Yoshiji H, Kojima H, et al. Leptin-mediated neovascularization is a prerequisite for progression of nonalcoholic steatohepatitis in rats. Hepatology. 2006;44:983-991. doi: 10.1002/hep.21338. 
25. Zou Y, Li J, Lu C, et al. High-fat emulsion-induced rat model of nonalcoholic steatohepatitis. Life Sci. 2006;79:1100-1107. doi: 10.1016/j.lfs.2006.03.021.

26. Deng QG, She H, Cheng JH, et al. Steatohepatitis induced by intragastric overfeeding in mice. Hepatology. 2005;42:905-914. doi:10.1002/hep.20877.

27. Sahai A, Malladi P, Pan X, et al. Obese and diabetic $\mathrm{db} / \mathrm{db}$ mice develop marked liver fibrosis in a model of nonalcoholic steatohepatitis: role of short-form leptin receptors and osteopontin. Am J Physiol Gastrointest Liver Physiol. 2004;287:G1035G1043. doi:10.1152/ajpgi.00199.2004.

28. Baffy G, Zhang CY, Glickman JN, Lowell BB. Obesity-related fatty liver is unchanged in mice deficient for mitochondrial uncoupling protein 2. Hepatology. 2002;35:753-761. doi: 10.1053/jhep.2002.32028.

29. Honda $\mathrm{H}$, Ikejima $\mathrm{K}$, Hirose $\mathrm{M}$, et al. Leptin is required for fibrogenic responses induced by thioacetamide in the murine liver. Hepatology. 2002;36:12-21. doi:10.1053/jhep.2002.33684.

30. Leclercq IA, Farrell GC, Schriemer R, Robertson GR. Leptin is essential for the hepatic fibrogenic response to chronic liver injury. J Hepatol. 2002;37:206-213. doi:10.1016/S0168-8278 (02)00102-2.

31. Lieber CS, Leo MA, Mak KM, et al. Model of nonalcoholic steatohepatitis. Am J Clin Nutr. 2004;79:502-509.

32. Kleiner DE, Brunt EM, Van Natta M, et al. Design and validation of a histological scoring system for nonalcoholic fatty liver disease. Hepatology. 2005;41:1313-1321. doi:10.1002/hep.20701.

33. Romestaing C, Piquet MA, Letexier D, et al. Mitochondrial adaptations to steatohepatitis induced by a methionine- and choline-deficient diet. Am J Physiol Endocrinol Metab. 2008; 294:E110-E119. doi:10.1152/ajpendo.00407.2007.

34. Fan JG, Farrell GC. VAT fat is bad for the liver, SAT fat is not! $J$ Gastroenterol Hepatol. 2008;23:829-832. doi:10.1111/j.14401746.2008.05474.x.

35. Fan JG, Li F, Cai XB, et al. Effects of nonalcoholic fatty liver disease on the development of metabolic disorders. $J$ Gastroenterol Hepatol. 2007;22:1086-1091. doi:10.1111/j.1440-1746. 2006.04781.x. 